\title{
Osteosarcopenia, the co-existence of osteoporosis and sarcopenia, is associated with social frailty in older adults
}

\author{
Tatsuro Inoue ${ }^{1,2} \cdot$ Keisuke Maeda $^{2}$ (1) $\cdot$ Shosuke Satake ${ }^{2,3} \cdot$ Yasumoto Matsui $^{4} \cdot$ Hidenori Arai $^{5}$
}

Received: 31 May 2021 / Accepted: 18 August 2021 / Published online: 7 September 2021

(c) The Author(s), under exclusive licence to Springer Nature Switzerland AG 2021

\begin{abstract}
Background Osteosarcopenia is a newly described, aging-associated condition. Social frailty is an important condition whose prevalence may have risen by physical distancing during the coronavirus disease 2019 pandemic. However, the relationship between these two remains unclear.

Aims To examine the association between osteosarcopenia and social frailty.

Methods This cross-sectional study was conducted using data from outpatients visiting general geriatric hospital frailty clinics. Bone mineral density (BMD) and muscle mass were measured using dual X-ray absorptiometry. Osteoporosis was defined as a BMD of $<70 \%$ of the young adult mean, according to the Japan Osteoporosis Society. Sarcopenia was diagnosed as per the Asian Working Group for Sarcopenia 2019 recommendation. Osteosarcopenia was defined as the co-existence of osteoporosis and sarcopenia. We defined social frailty using a questionnaire comprising four items: general resources, social resources, social behavior, and basic social needs. Ordinal logistic regression analysis was performed with social frailty status and osteosarcopenia as the dependent and independent variables, respectively.

Results We included 495 patients (mean age $=76.5 \pm 7.2$ years) in the analysis; of these, $58.2 \%$ were robust and $17.2 \%, 13.5 \%$, and $11.1 \%$ had osteoporosis alone, sarcopenia alone, and osteosarcopenia, respectively. Social frailty prevalence increased stepwise from $8.0 \%$ in robust patients to $11.8 \%, 17.9 \%$, and $29.1 \%$ among those with osteoporosis alone, sarcopenia alone, and osteosarcopenia, respectively $(P<0.001)$. Logistic regression analysis revealed that only osteosarcopenia was significantly associated with social frailty (pooled odds ratio: 2.117 ; $95 \%$ confidence interval: $1.104-4.213$ ).
\end{abstract}

Discussion Comprehensive assessment of osteosarcopenia and social frailty is needed for disability prevention in older adults.

Keywords Osteosarcopenia $\cdot$ Osteoporosis $\cdot$ Sarcopenia $\cdot$ Social frailty

\section{Introduction}

Keisuke Maeda

kskmaeda1701@gmail.com

1 Department of Physical Therapy, Niigata University of Health and Welfare, 1398 Shimami-cho, Kita-ku, Niigata 950-3198, Japan

2 Department of Geriatric Medicine, Hospital, National Center for Geriatrics and Gerontology, 7-430 Morioka, Obu, Aichi 474-8511, Japan

3 Department of Frailty Research, Research Institute, National Center for Geriatrics and Gerontology, 7-430 Morioka, Obu, Aichi 474-8511, Japan

4 Center for Frailty and Locomotive Syndrome, Hospital, National Center for Geriatrics and Gerontology, 7-430 Morioka, Obu, Aichi 474-8511, Japan

5 National Center for Geriatrics and Gerontology, 7-430 Morioka, Obu, Aichi 474-8511, Japan
Osteoporosis and sarcopenia are common geriatric diseases. Osteoporosis is a condition of decreased bone mineral density (BMD) [1] and is strongly associated with osteoporotic fractures [2]. Sarcopenia is a skeletal muscle disease associated with decreased muscle strength, muscle mass, and physical function [3] as well as with falls, disability, diseases, hospitalization, and death [4]. Bones and muscles have strong interactions [5], and their structures and functions decline in parallel with aging [6]. Therefore, considering osteoporosis and sarcopenia simultaneously is key to preventing loss of independent life.

Osteosarcopenia is a newly described syndrome that entails the co-existence of osteoporosis and sarcopenia [7]. It is reportedly associated with chronic diseases $[8,9]$, the endocrine system $[10,11]$, frailty $[8,12]$, nutritional status 
[13], and physical function [14]. While osteosarcopenia is associated with declining physical function, sarcopenia and osteoporosis independently are not always associated with physical function in community-dwelling older adults [15]. Thus, osteoporosis and sarcopenia may have additive or synergistic effects that may contribute to negative health outcomes.

Frailty is one of the commonest geriatric syndromes causing physical, psychological, and social challenges in older adults [16]. Physical frailty indicates physical vulnerability [17], whereas social frailty reflects loneliness, economic burden, and reduced social participation [18]. One study reported a nearly $30 \%$ overlap between physical and social frailty [19]. Social frailty is a risk factor for longterm mortality and disability [19], and has a prevalence of approximately 18-30\% among community-dwelling older adults $[19,20]$. Furthermore, social frailty leads to physical frailty [21]. Social frailty is an important condition, because it is common among older adults and results in a loss of independency in activities of daily living. Research on social frailty is important, particularly for situations such as quarantines or isolations, which have recently arisen due to the coronavirus disease 2019 pandemic [22].

Osteosarcopenia and social frailty are commonly associated with falls [23, 24] and fractures [23, 25]; however, the relationship between these two conditions has not been elucidated. Identifying the association between osteosarcopenia and social frailty may help develop effective intervention strategies for improved health and longevity and for preventing disability. Therefore, the present study aimed to examine the association between osteosarcopenia and social frailty in older patients who visited a frailty clinic.

\section{Methods}

\section{Participants}

This cross-sectional study was conducted among outpatients who received consultation at a frailty clinic in a general geriatric hospital in Aichi (Obu, Japan). We extracted data from the Frailty Registry Study, which involved outpatients visiting the frailty clinics in the National Center for Geriatrics and Gerontology, Japan, between June 2017 and July 2019. The physical composition and function were evaluated during the patient's first clinical visit. The inclusion criteria were age $\geq 65$ years and the ability to walk independently. The exclusion criteria were as follows: (1) presence of visual or hearing impairment that interfered with daily life, (2) presence of obvious tetraplegia, (3) previous diagnosis of dementia (including prescription of medications for dementia), (4) progressive functional decline of the extremities (e.g., Parkinson's disease), (5) being in the terminal stage of life, and (6) being deemed unsuitable for study registration by the attending physician.

This study was conducted in accordance with the 1964 Declaration of Helsinki and received approval from the Ethics Committees of the National Center for Geriatrics and Gerontology (No. 1459) and the Niigata University of Health and Welfare (No. 18521-201124). We obtained written informed consent from the patients for registration in the Frailty Registry Study.

\section{Osteosarcopenia}

Osteosarcopenia was defined as the co-existence of osteoporosis and sarcopenia. BMD and muscle mass were measured using dual X-ray absorptiometry (Lunar iDXA; GE Healthcare, Chicago, IL). BMD was measured at the femoral neck and at L2-L4; the lower value was adopted. Muscle mass was expressed using the skeletal muscle index (SMI), which was calculated as the sum of the limb skeletal muscle mass divided by the square of height $(\mathrm{m})$.

Osteoporosis was defined by a BMD of $<70 \%$ of the young adult mean (YAM) (according to the Japan Osteoporosis Society criteria) [26]. Based on the World Health Organization criteria, which are widely used for the diagnosis of osteoporosis, this is almost equivalent to a BMD of $\leq 2.5$ standard deviations below the YAM [27].

Sarcopenia was diagnosed as per the Asian Working Group for Sarcopenia (AWGS) 2019 consensus [28]. Calf circumferences (male: $<34 \mathrm{~cm}$, female $<33 \mathrm{~cm}$ ) were used to screen for sarcopenia, and low muscle mass was defined by an SMI $<7.0 \mathrm{~kg} / \mathrm{m}^{2}$ for males and $<5.4 \mathrm{~kg} / \mathrm{m}^{2}$ for females [28]. Decreased physical function was defined as follows: gait speed $<1.0 \mathrm{~m} / \mathrm{s}$, five-time chair stand test (5CST) time $\geq 12 \mathrm{~s}$, and short physical performance battery (SPPB) score $\leq 9$ points [28]. The gait speed is measured as the comfortable walking speed on a 4-m flat floor [29]. The 5CST time is the time taken to stand up from a seated position and sit down as quickly as possible for five times [30]; patients were instructed to cross their upper limbs in front of their chests and not support them on armrests. The SPPB is a physical function test comprising the standing balance, gait speed, and 5CST time [31]; it is scored on a scale of $0-12$ points, with higher scores indicating better physical functioning. Muscle strength was assessed using the handgrip strength: patients were evaluated in a standing position with their upper limbs along the sides of their trunk. Muscle weakness was defined by a handgrip strength $<28 \mathrm{~kg}$ for males and $<18 \mathrm{~kg}$ for females [28]. Patients scheduled for orthopedic surgery (hip or knee arthroplasty: $n=115$ ) were diagnosed as having sarcopenia based on their muscle mass and strength, because hip or knee osteoarthritis may affect the physical function. 


\section{Social frailty}

We defined social frailty using the questionnaire reported by Yamada et al. [19], which is based on Bunt's social frailty concept [32]. It comprised four items, namely general resources, social resources, social behavior, and basic social needs.

1. For general resources, we asked the patients, "Please tell us about your financial condition." The options included: (a) "I have enough to help others", (b) "I don't mind living my life", (c) "I need some help", and (d) "I need full assistance". Answer (d) was considered indicative of "poor general resources" and awarded 1 point.

2. For social resources, we asked the patients, "Do you live with anyone?". A response of "living alone" was considered indicative of "poor social resources" and awarded 1 point.

3. For social behavior, we asked the patients whether they participated in the following activities: (a) local festivals or events, (b) community activities in the neighborhood, (c) self-management groups, and (d) volunteering activities. Non-participation in any of these was defined as "poor social behavior" and awarded 1 point.

4. Regarding basic social needs, we asked the patients about their relationships with their neighbors. The options included: (a) "I have neighbors who go to each other's houses," (b) "I have neighbors with whom I talk privately," (c) "I have neighbors that I occasionally meet and talk to," and (d) "I don't have any neighbors to talk to". Answer (d) was considered indicative of "poor basic social needs" and awarded 1 point.

The total score was calculated from the four items: 0,1 , and $\geq 2$ points were defined as being socially robust, socially prefrail, and social frail, respectively [19].

\section{Other variables}

From the registry, we extracted data on other factors, including age, gender, body mass index (BMI), number of comorbidities and medications, medications for osteoporosis, educational history, mini nutritional assessment-short form (MNA-SF) score [33], mini-mental state examination (MMSE) score [34], and presence of physical frailty [17]. The MNA-SF is a nutritional screening tool, with scores ranging from 0 to 14 points [33]. The MMSE is a screening test of cognitive function, with scores ranging from 0 to 30 points [34]. Physical frailty was assessed using the Japanese version of the cardiovascular health study criteria [35], which are based on the frailty phenotype [17]. Physical frailty was defined as the presence of three or more of the following symptoms: shrinking, weakness, exhaustion, slowness, and low activity [35].

\section{Sample size calculation}

A previous study reported that among older adults, the incidences of muscle mass loss with and without social frailty were approximately $60 \%$ and $40 \%$, respectively [36]. We assumed that the ratio of adults with osteosarcopenia to adults without osteosarcopenia was approximately 1:4 [14]. Sample sizes were calculated using the Power and Sample Size Calculation software, version 3.1.2 0 (Department of Biostatistics, Vanderbilt University, Nashville, TN); at a power of 0.8 and an alpha of 0.05 (two-sided), the current study required at least 300 participants.

\section{Statistical analysis}

Patients were categorized as having social robust, osteoporosis alone, sarcopenia alone, and osteosarcopenia. The patient characteristics were compared between the four groups. Continuous variables were compared using one-way analysis of variance for parametric variables and the Kruskal-Wallis test for non-parametric variables. The post hoc tests were the Tukey-Kramer and Steel-Dwass tests, used as appropriate. The Fisher's exact test was used to compare categorical variables. The proportion of social frailty in the four groups was analyzed using the Cochrane-Armitage trend test.

To investigate the association between osteosarcopenia and social frailty, we performed ordinal logistic regression analysis with social frailty (robust, social prefrailty, frailty) as the dependent variable and osteosarcopenia as the independent variable. We included variables that were significantly different in the four groups of potential confounders. Multicollinearity between explanatory variables was determined using the variance inflation factor (VIF).

We also compared the proportion of patients with severely decreased BMD or low sarcopenia-related indicators (muscle mass, muscle strength, and physical function) in the four groups using the Cochrane-Armitage trend test. Patients with osteoporosis were categorized into two groups having mildly and severely decreased BMD using the median. Furthermore, patients were also categorized into three groups on the basis of sarcopenia-related indicators: normal (indicators above the AWGS 2019-recommended cut-off values) and mild and severe (indicators below the cut-off value, divided by the median). We performed all statistical analyses using SPSS version 26.0 (IBM). A $P$ value of $<0.05$ was considered statistically significant.

\section{Results}

Of the 499 patients in the registry, 4 with missing values were excluded; therefore, 495 patients were included in the analysis. Among these, 288 (58.2\%) patients were robust 
and 85 (17.2\%), 67 (13.5\%), and 55 (11.1\%) patients had osteoporosis alone, sarcopenia alone, and osteosarcopenia, respectively. Compared to patients who were robust, patients with osteosarcopenia were significantly older and had lower BMI, MNA-SF scores, and MMSE scores. Osteoporosis medication usage was the lowest among those with osteosarcopenia (Table 1).

Regarding the social frailty status, 216 (43.6\%), 218 $(44.0 \%)$, and $61(12.3 \%)$ patients were socially robust, socially prefrail, and socially frail, respectively. The prevalence of social frailty increased stepwise from $8.0 \%$ among robust patients to $11.8 \%, 17.9 \%$, and $29.1 \%$ among those with osteoporosis alone, sarcopenia alone, and osteosarcopenia, respectively $(P<0.001)$ (Fig. 1$)$.

Regarding the social frailty components, financial difficulties had the lowest proportion and lack of social activity had the highest proportion (Table 2).

The results of the ordinal logistic regression analysis for the social frailty status are shown in Fig. 2. Osteoporosis alone and sarcopenia alone were not associated with social frailty. Conversely, osteosarcopenia was significantly associated with social frailty (pooled odds ratio: 2.117; 95\% confidence interval: 1.104-4.213). The VIF among the explanatory variables was $<2.0$.

Figure 3 shows the results of comparisons between groups with severely decreased BMD and low sarcopeniarelated indicators. There were significantly more patients with osteosarcopenia than osteoporosis alone in the group with severely decreased BMD $(P=0.022)$ (Fig. 3a). Conversely, the proportion of patients with severely reduced sarcopenia-related indications did not differ between patients with osteosarcopenia and sarcopenia for any sarcopeniarelated indicators (Fig. 3b).

\section{Discussion}

Our study uncovered two novel findings. First, osteosarcopenia was significantly associated with social frailty, although osteoporosis and sarcopenia alone were not. Second, osteosarcopenia was not associated with the severity of sarcopenia, but affected more patients with a severely decreased

Table 1 Demographic characteristics of the study participants

\begin{tabular}{|c|c|c|c|c|c|c|}
\hline & $\begin{array}{l}\text { Overall } \\
(N=495)\end{array}$ & $\begin{array}{l}\text { Robust } \\
(N=288)\end{array}$ & $\begin{array}{l}\text { Osteoporosis } \\
\text { alone } \\
(N=85)\end{array}$ & $\begin{array}{l}\text { Sarcopenia } \\
\text { alone } \\
(N=67)\end{array}$ & $\begin{array}{l}\text { Osteosarcopenia } \\
(N=55)\end{array}$ & $P$ value \\
\hline Age, years, mean (SD) & $76.5(7.2)$ & $74.9(7.2)$ & $77.2(6.1)^{* a}$ & $80.0(6.2)^{* * a}$ & $78.9(8.1)^{* * a}$ & $<0.001$ \\
\hline Women, $n(\%)$ & $340(68.7)$ & $200(69.7)$ & $75(90.4)$ & $25(36.8)$ & $40(70.2)$ & $<0.001$ \\
\hline BMI, mean (SD) & $23.7(4.3)$ & $25.2(4.2)$ & $23.7(3.4)^{* * a}$ & $20.9(2.4)^{* * a, b}$ & $19.2(2.9)^{* * a, b}$ & $<0.001$ \\
\hline Education, years, median (IQR) & $12(9-12)$ & $12(9-14)$ & $12(9-12)^{* a}$ & $12(9-12)$ & $11(9-12)$ & 0.001 \\
\hline Number of comorbidities, $N(\%)$ & & & & & & 0.694 \\
\hline 0 & $17(3.4)$ & $13(4.5)$ & $2(2.4)$ & $1(1.5)$ & $1(1.8)$ & \\
\hline $1-3$ & $207(41.9)$ & $125(43.6)$ & $34(40.0)$ & $26(38.8)$ & $22(40.0)$ & \\
\hline$\geq 4$ & $270(54.7)$ & $149(51.9)$ & $49(57.6)$ & $40(59.7)$ & $32(58.2)$ & \\
\hline Number of medications, $N(\%)$ & & & & & & 0.061 \\
\hline 0 & $39(7.9)$ & $27(9.4)$ & $6(7.1)$ & $3(4.5)$ & $3(5.5)$ & \\
\hline $1-4$ & $185(37.4)$ & $115(39.9)$ & $34(40.0)$ & $15(22.4)$ & $21(38.2)$ & \\
\hline$\geq 5$ & $271(54.7)$ & $146(50.7)$ & $45(52.9)$ & $49(73.1)$ & $31(56.4)$ & \\
\hline Medication for osteoporosis, $N(\%)$ & $36(7.3)$ & $17(5.9)$ & $8(9.4)$ & $10(14.9)$ & $1(1.8)$ & 0.025 \\
\hline MNA-SF, median (IQR) & $13(11-14)$ & $13(12-14)$ & $12(11-14)$ & $12(10-13)^{* * a}$ & $11(9-12)^{* * a, * c}$ & $<0.001$ \\
\hline MMSE, median (IQR) & $27(25-29)$ & $28(26-30)$ & $27(25-29)$ & $26(23-28)^{* * a}$ & $26(24-28)^{* * a}$ & $<0.001$ \\
\hline Physical frailty, $N(\%)$ & $154(31.1)$ & $60(20.8)$ & $34(40.0)$ & $28(41.8)$ & $32(58.2)$ & $<0.001$ \\
\hline
\end{tabular}

Statistical analysis: one-way analysis of variance for parametric variables, the Kruskal-Wallis test for non-parametric variables, and the Fisher's exact test for categorical variables. Post-hoc tests: the Tukey-Kramer test for parametric variables, the Steel-Dwass test for non-parametric variables

$S D$ standard deviation, BMI body mass index, IQR interquartile rage, MNA-SF Mini Nutritional Assessment-short form, $M M S E$ Mini-Mental State Examination

$* P<0.05$

$* * P<0.01$

${ }^{\text {a }}$ Significant differences: compared with robust

${ }^{\mathrm{b}}$ Compared with osteoporosis alone

${ }^{\mathrm{c} C o m p a r e d}$ with sarcopenia alone 
Fig. 1 The prevalence of social frailty in osteosarcopenia. $P<0.001$ for social frailty using the Cochrane-Armitage trend test

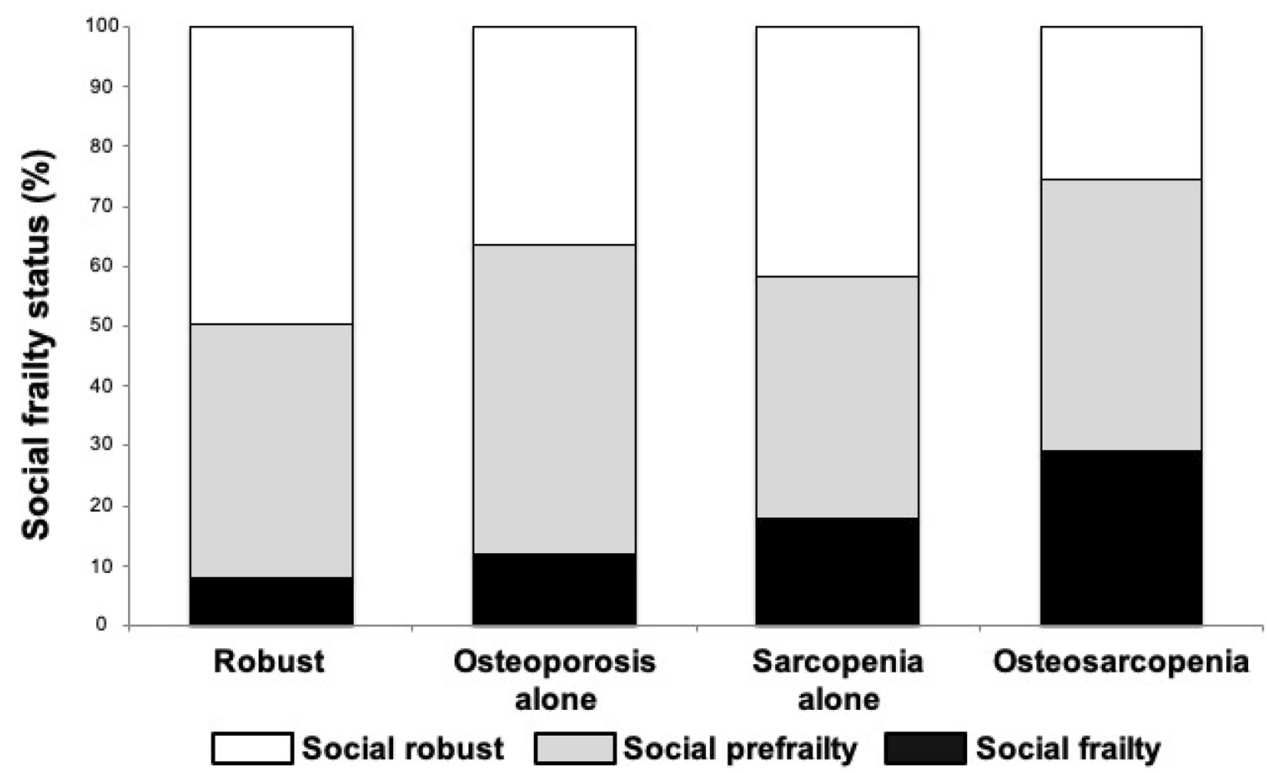

Table 2 The association between osteosarcopenia and social frailty components

\begin{tabular}{lllllrr}
\hline & Overall & Robust & Osteoporosis alone & Sarcopenia alone & Osteosarcopenia & $P$ value \\
\hline Social frailty component & & & & & \\
$\quad$ Financial difficulties & $1(0.2)$ & $0(0)$ & $0(0)$ & $1(1.5)$ & $0(0)$ & 0.246 \\
Influential contact with neighbors & $41(8.3)$ & $15(5.2)$ & $3(3.5)$ & $10(14.9)$ & $13(23.6)$ & $8(14.5)$ \\
Living alone & $77(15.6)$ & $43(14.9)$ & $20(23.5)$ & $6(9.0)$ & $35(52.2)$ & $38(69.1)$ \\
Lack of social activity & $224(45.3)$ & $110(38.2)$ & $41(48.2)$ & 0.091 \\
\hline
\end{tabular}

Statistical analysis: the Fisher's exact test

Fig. 2 Multivariate analyses for social frailty status. Ordinal logistic regression analysis for social frailty status. Osteosarcopenia showed a significant association with the social frailty status (pooled odds ratio: 2.117; 95\% confidence interval: 1.064-4.213)

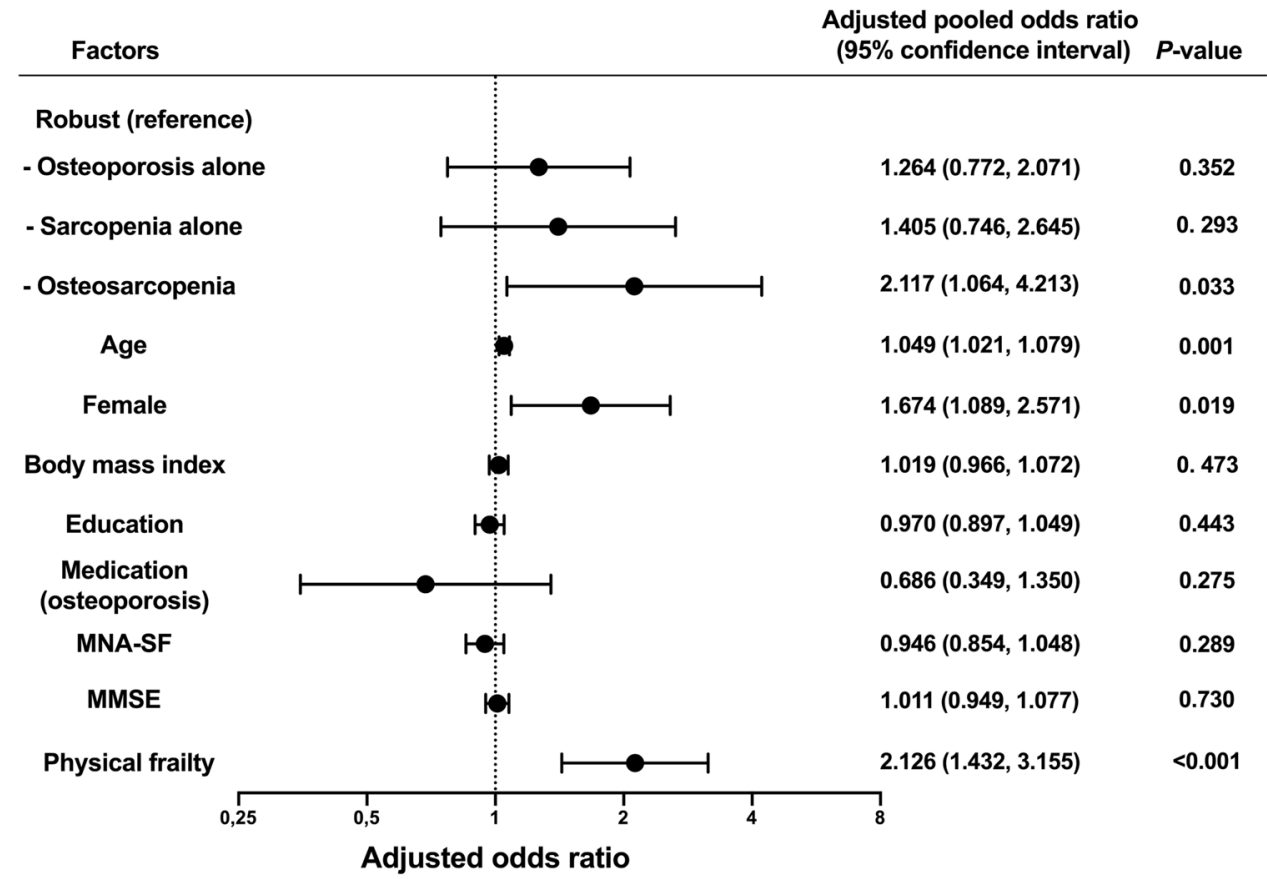


Fig. 3 Comparison of the severity of osteoporosis and sarcopenia-related indicators according to the osteosarcopenia status. a Osteoporosis-related indicators. Using the median value, the bone mineral density of patients with osteoporosis was categorized into two groups (mildly and severely decreased groups). b Sarcopenia-related indicators. Patients were categorized into three groups: normal (above the AWGS 2019-recommended cut off value) and mild and severe (divided by the median among those with values below the cut off). $* P=0.022$ for patients with severely reduced BMD, using the Cochrane-Armitage trend test. $O P$ osteoporosis alone, $O S$ osteosarcopenia, $S A$ sarcopenia alone, $5 C S T$ five-time chair stand test, $S P P B$ short physical performance battery $\mathbf{a}$

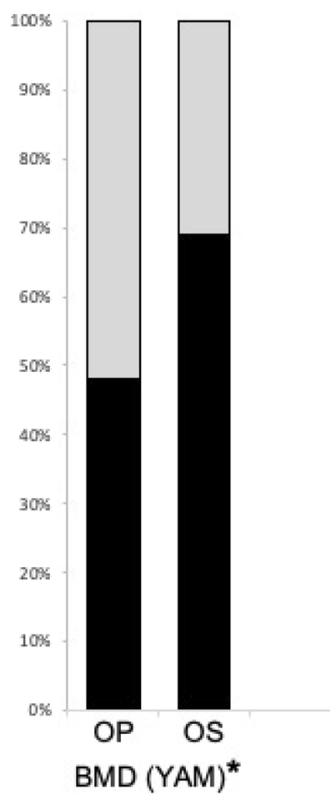

b

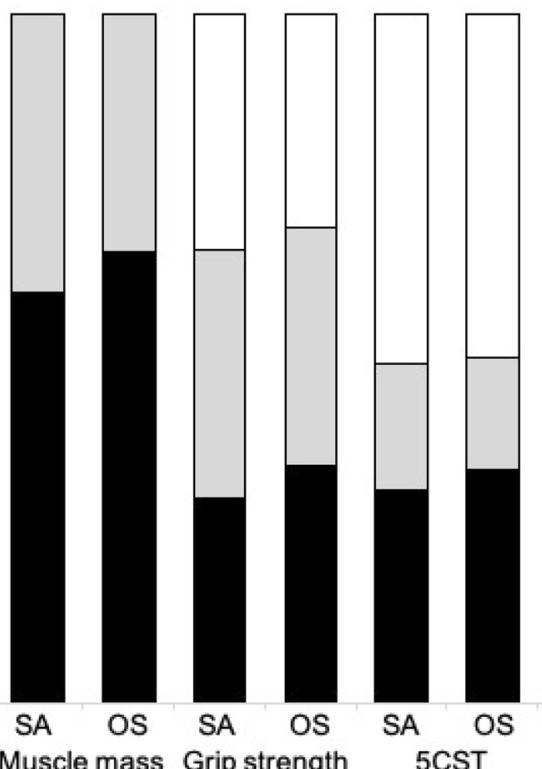

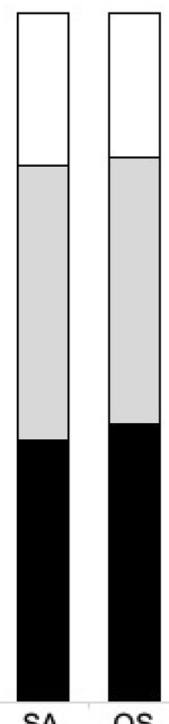

SA OS

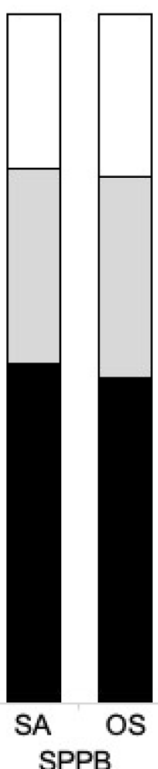

Normal
BMD. Our findings clarify the relationship between osteosarcopenia and social frailty and also elucidate some of the pathogenesis of osteosarcopenia.

We found that osteosarcopenia was associated with social frailty; a cross-sectional association between sarcopeniarelated indicators (such as muscle weakness) and social frailty has previously been reported in community-dwelling older adults [20]. An association between sarcopenia and activity restriction has also been reported [37]. The results of these previous studies suggest that osteosarcopenia, potentially within sarcopenia, may be strongly associated with social frailty. None of the studies has shown a causal relationship between osteosarcopenia and social frailty, although several studies have demonstrated that social isolation and loneliness lead to physical inactivity [38, 39]. Because physical inactivity causes atrophy of the bone and muscle tissues [40], which in turn may lead to the development and progression of sarcopenia and osteoporosis [41, 42], it is reasonable to assume that social frailty contributes to osteosarcopenia. Regarding interventions for social frailty, participation in communities that promote social interactions among older residents has been reported to reduce the risk of disability [43]. Activities, such as joining a health-promoting exercise group, may promote behavioral changes [44] and prevent osteosarcopenia. The reverse causality assumption would be that osteosarcopenia is the cause of social frailty. However, to our knowledge, only few studies have examined this.

Osteosarcopenia is a new concept and remains controversial, as its definition slightly differs across studies. Some studies have included osteopenia in the definition of osteosarcopenia [10, 23]. However, we included osteoporosis in the definition of osteosarcopenia, because it is more reasonable to show the impact of osteosarcopenia, rather than of sarcopenia as a skeletal muscle disease or osteoporosis as a bone disease, on the patient outcomes. Notably, it has been consistently reported that osteosarcopenia entails the co-existence of a low BMD and low muscle mass, muscle strength, and physical function; thus, osteoporosis and sarcopenia may have additive or synergistic effects leading to negative health outcomes. In a cross-sectional study by Huo et al. [45], osteosarcopenia was associated with more selfreported past falls and fractures as compared to sarcopenia and osteoporosis alone. Although longitudinal studies are needed to clarify the impact of osteosarcopenia on the adverse outcomes, we believe that the co-existence of osteoporosis and sarcopenia, which is common in older adults, may be an important point to consider for preventing future disabilities and extending longevity.

There is no golden standard definition of social frailty. The definition by Yamada et al. [19], which we adopted in this study, is based on the concept of social frailty put forth by Bunt et al. [32]. This concept identified four social factors necessary for maintaining the well-being of older adults: general resources, social resources, social behavior, and basic social needs. Yamada et al.'s definition encompasses all four factors, and the absence of any one impairs the wellbeing, leading to disability and mortality [19]. The developed definition of social frailty implicates it as a risk factor for negative health outcomes and is necessary for validating 
the definition. Hence, we believe that the definition of Yamada et al. that we have adopted is worthy of use. Meanwhile, some new criteria for social frailty have been reported [46], and the concept needs to be unified in future studies.

Another novel finding of our study was that osteosarcopenia was not associated with sarcopenia severity, i.e., the skeletal muscle mass, muscle strength, and physical function; however, it affected more patients with a severely decreased BMD. Nonetheless, the causal relationship between social frailty and osteoporosis (as well as osteoporotic fractures) remains unclear. Social frailty has previously been reported to be associated with cardiovascular diseases [47], dementia [48], and disability [49]. Conversely, patients with hip fractures have been reported to exhibit a high rate of social isolation prior to the fractures [25,50], and longitudinal studies have reported that solitary living and social isolation are risk factors for falls [24] and fall-related injuries [51]. Therefore, it is possible that social frailty leads to the progression of osteoporosis through decreased physical activity [52]. In this study, the population included may be the reason why osteosarcopenia was not associated with sarcopenia severity: we used a registry that enrolled ambulatory outpatients with independent activities of daily living, which may have resulted in the lower prevalence of a severe decrease in the sarcopenia-related indicators.

There are several limitations to our study. Our study shows a cross-sectional association between osteosarcopenia and social frailty, and the causal relationship between the two is yet to be elucidated; longitudinal studies must clarify this. Furthermore, a reciprocal interaction between osteosarcopenia and social frailty should also be noted. Second, any generalization of this study's findings needs to be carefully re-evaluated because we included outpatients with untreated osteoporosis attending a frailty clinic. Third, we could not examine which factors contributed to a closer association between social frailty and osteosarcopenia. Finally, this study included outpatients who visited a frailty clinic; thus, we need to be careful while generalizing the study findings to community-dwelling older adults.

\section{Conclusions}

We found that osteoporosis or sarcopenia alone was not associated with social frailty, whereas osteosarcopenia was significantly associated with social frailty. Furthermore, while osteosarcopenia was not associated with the severity of sarcopenia, it affected more patients with a severely decreased BMD. Further studies are needed to clarify the causal relationship between osteosarcopenia and social frailty for improved health and longevity and for prevention of disability in older adults.
Author contributions All authors have read the manuscript and approved its submission. TI, KM, SS, YM, and HA contributed to study conceptualization and design. All authors contributed to the acquisition, analysis, and interpretation of data. TI, KM, SS, YM, and HA drafted the manuscript. All authors contributed to critical revisions of the manuscript for important intellectual content. TI and KM were responsible for statistical analyses. KM supervised the study.

Funding This study was supported by the Research Funding for Longevity Sciences (20-57) from the National Center for Geriatrics and Gerontology, Japan.

Availability of data and materials The datasets generated during and/or analysed during the current study are available from the corresponding author on reasonable request.

Code availability Not applicable.

\section{Declarations}

Conflict of interest The authors have no conflicts of interest to declare.

Ethical approval This study was conducted in accordance with the 1964 Declaration of Helsinki and with the approval of the Ethics Committees of the National Center for Geriatrics and Gerontology (No. 1459) and Niigata University of Health and Welfare (No. 18521-201124).

Informed consent We obtained written informed consent from the patients for registration in the Frail Registry Study.

\section{References}

1. Kanis JA, Adachi JD, Cooper C et al (2013) Standardising the descriptive epidemiology of osteoporosis: recommendations from the Epidemiology and Quality of Life Working Group of IOF. Osteoporos Int 24:2763-2764. https://doi.org/10.1007/ s00198-013-2413-7

2. Kanis JA (1994) Assessment of fracture risk and its application to screening for postmenopausal osteoporosis: synopsis of a WHO report. WHO Study Group Osteoporos Int 4:368-381. https://doi. org/10.1007/BF01622200

3. Cao L, Morley JE (2016) Sarcopenia is recognized as an independent condition by an international classification of disease, tenth revision, clinical modification (ICD-10-CM) code. J Am Med Dir Assoc 17:675-677. https://doi.org/10.1016/j.jamda.2016. 06.001

4. Cruz-Jentoft AJ, Sayer AA (2019) Sarcopenia. Lancet 393:26362646. https://doi.org/10.1016/S0140-6736(19)31138-9

5. Schoenau E, Fricke O (2006) Interaction between muscle and bone. Horm Res 66:73-78. https://doi.org/10.1159/000096626

6. Locquet M, Beaudart C, Durieux N et al (2019) Relationship between the changes over time of bone and muscle health in children and adults: a systematic review and meta-analysis. BioRxiv. https://doi.org/10.1101/541524

7. Hirschfeld HP, Kinsella R, Duque G (2017) Osteosarcopenia: where bone, muscle, and fat collide. Osteoporos Int 28:27812790. https://doi.org/10.1007/s00198-017-4151-8

8. Wang YJ, Wang Y, Zhan JK et al (2015) Sarco-osteoporosis: prevalence and association with frailty in Chinese communitydwelling older adults. Int J Endocrinol 2015:482940. https://doi. org/10.1155/2015/482940 
9. Huo YR, Suriyaarachchi P, Gomez F et al (2015) Phenotype of osteosarcopenia in older individuals with a history of falling. J Am Med Dir Assoc 16:290-295. https://doi.org/10.1016/j. jamda.2014.10.018

10. Suriyaarachchi P, Gomez F, Curcio CL et al (2018) High parathyroid hormone levels are associated with osteosarcopenia in older individuals with a history of falling. Maturitas 113:21-25. https://doi.org/10.1016/j.maturitas.2018.04.006

11. Poggiogalle E, Cherry KE, Su LJ et al (2019) Body composition, IGF1 status, and physical functionality in nonagenarians: implications for osteosarcopenia. J Am Med Dir Assoc 20:7075.e2. https://doi.org/10.1016/j.jamda.2018.07.007

12. Okamura H, Ishikawa K, Kudo $\mathrm{Y}$ et al (2020) Risk factors predicting osteosarcopenia in postmenopausal women with osteoporosis: a retrospective study. PLoS ONE 15:e0237454. https:// doi.org/10.1371/journal.pone.0237454

13. Reiss J, Iglseder B, Alzner R et al (2019) Sarcopenia and osteoporosis are interrelated in geriatric inpatients. Z Gerontol Geriatr 52:688-693. https://doi.org/10.1007/s00391-019-01553-z

14. Sepúlveda-Loyola W, Phu S, Bani Hassan E et al (2020) The joint occurrence of osteoporosis and sarcopenia (osteosarcopenia): definitions and characteristics. J Am Med Dir Assoc 21:220-225. https://doi.org/10.1016/j.jamda.2019.09.005

15. Drey M, Sieber CC, Bertsch T et al (2016) Osteosarcopenia is more than sarcopenia and osteopenia alone. Aging Clin Exp Res 28:895-899. https://doi.org/10.1007/s40520-015-0494-1

16. Rockwood K (2005) What would make a definition of frailty successful. Age Ageing 34:432-434. https://doi.org/10.1093/ ageing/afi146

17. Fried LP, Tangen CM, Walston $\mathbf{J}$ et al (2001) Frailty in older adults: evidence for a phenotype. J Gerontol A Biol Sci Med Sci 56:M146-M156. https://doi.org/10.1093/gerona/56.3.m146

18. Makizako H, Shimada H, Tsutsumimoto K et al (2015) Social frailty in community-dwelling older adults as a risk factor for disability. J Am Med Dir Assoc 16:1003.e7-11. https://doi.org/ 10.1016/j.jamda.2015.08.023

19. Yamada M, Arai H (2018) Social frailty predicts incident disability and mortality among community-dwelling japanese older adults. J Am Med Dir Assoc 19:1099-1103. https://doi.org/10. 1016/j.jamda.2018.09.013

20. Tsutsumimoto K, Doi T, Makizako H et al (2018) Social frailty has a stronger impact on the onset of depressive symptoms than physical frailty or cognitive impairment: a 4-year follow-up longitudinal cohort study. J Am Med Dir Assoc 19:504-510. https://doi.org/10.1016/j.jamda.2018.02.008

21. Makizako H, Shimada H, Doi T et al (2018) Social frailty leads to the development of physical frailty among physically nonfrail adults: a four-year follow-up longitudinal cohort study. Int J Environ Res Public Health 15:E490. https://doi.org/10.3390/ ijerph 15030490

22. Lewnard JA, Lo NC (2020) Scientific and ethical basis for social-distancing interventions against COVID-19. Lancet Infect Dis 20:631-633. https://doi.org/10.1016/S1473-3099(20) 30190-0

23. Salech F, Marquez C, Lera L et al (2021) Osteosarcopenia predicts falls, fractures, and mortality in chilean community-dwelling older adults. J Am Med Dir Assoc 22:853-858. https://doi.org/10. 1016/j.jamda.2020.07.032

24. Pohl JS, Cochrane BB, Schepp KG et al (2018) Falls and social isolation of older adults in the national health and aging trends study. Res Gerontol Nurs 11:61-70. https://doi.org/10.3928/19404 921-20180216-02

25. Smith TO, Dainty JR, MacGregor A (2018) Trajectory of social isolation following hip fracture: an analysis of the English longitudinal study of ageing (ELSA) cohort. Age Ageing 47:107-112. https://doi.org/10.1093/ageing/afx129
26. Soen S, Fukunaga M, Sugimoto T et al (2013) Diagnostic criteria for primary osteoporosis: year 2012 revision. J Bone Miner Metab 31:247-257. https://doi.org/10.1007/s00774-013-0447-8

27. WHO (1994) Assessment of fracture risk and its application to screening for postmenopausal osteoporosis. Report of a WHO Study Group. World Health Organ Tech Rep Ser 843:1-129

28. Chen LK, Woo J, Assantachai P et al (2020) Asian working group for sarcopenia: 2019 consensus update on sarcopenia diagnosis and treatment. J Am Med Dir Assoc 21:300-307.e2. https://doi. org/10.1016/j.jamda.2019.12.012

29. Makizako H, Nakai Y, Tomioka K et al (2020) Effects of a multicomponent exercise program in physical function and muscle mass in sarcopenic/pre-sarcopenic adults. J Clin Med 9:E1386. https://doi.org/10.3390/jcm9051386

30. Whitney SL, Wrisley DM, Marchetti GF et al (2005) Clinical measurement of sit-to-stand performance in people with balance disorders: validity of data for the five-times-sit-to-stand test. Phys Ther 85:1034-1045. https://doi.org/10.1093/ptj/85.10.1034

31. Guralnik JM, Ferrucci L, Pieper CF et al (2000) Lower extremity function and subsequent disability: consistency across studies, predictive models, and value of gait speed alone compared with the short physical performance battery. J Gerontol A Biol Sci Med Sci 55:M221-M231. https://doi.org/10.1093/gerona/55.4.m221

32. Bunt S, Steverink N, Olthof J et al (2017) Social frailty in older adults: a scoping review. Eur J Ageing 14:323-334. https://doi. org/10.1007/s10433-017-0414-7

33. Rubenstein LZ, Harker JO, Salvà A et al (2001) Screening for undernutrition in geriatric practice: developing the short-form mini-nutritional assessment (MNA-SF). J Gerontol A Biol Sci Med Sci 56:M366-M372. https://doi.org/10.1093/gerona/56.6. m366

34. Folstein MF, Folstein SE, McHugh PR (1975) "Mini-mental state". A practical method for grading the cognitive state of patients for the clinician. J Psychiatr Res 12:189-198. https://doi. org/10.1016/0022-3956(75)90026-6

35. Satake S, Arai H (2020) Chapter 1 Frailty: Definition, diagnosis, epidemiology. Geriatr Gerontol Int 20 (Suppl 1):7-13. https://doi. org/10.1111/ggi.13830

36. Makizako H, Kubozono T, Kiyama R et al (2019) Associations of social frailty with loss of muscle mass and muscle weakness among community-dwelling older adults. Geriatr Gerontol Int 19:76-80. https://doi.org/10.1111/ggi.13571

37. Merchant RA, Chen MZ, Wong BLL et al (2020) Relationship between fear of falling, fear-related activity restriction, frailty, and sarcopenia. J Am Geriatr Soc 68:2602-2608. https://doi.org/ $10.1111 /$ jgs. 16719

38. Kobayashi LC, Steptoe A (2018) Social isolation, loneliness, and health behaviors at older ages: longitudinal cohort study. Ann Behav Med 52:582-593. https://doi.org/10.1093/abm/kax033

39. Hawkley LC, Thisted RA, Cacioppo JT (2009) Loneliness predicts reduced physical activity: cross-sectional \& longitudinal analyses. Health Psychol 28:354-363. https://doi.org/10.1037/a0014400

40. Kirk B, Al Saedi A, Duque G (2019) Osteosarcopenia: a case of geroscience. Aging Med 2:147-156. https://doi.org/10.1002/ agm2.12080

41. Warburton DE, Nicol CW, Bredin SS (2006) Health benefits of physical activity: the evidence. CMAJ 174:801-809. https://doi. org/10.1503/cmaj.051351

42. Montero-Fernández N, Serra-Rexach JA (2013) Role of exercise on sarcopenia in the elderly. Eur J Phys Rehabil Med 49:131-143

43. Hikichi H, Kondo N, Kondo K et al (2015) Effect of a community intervention programme promoting social interactions on functional disability prevention for older adults: propensity score matching and instrumental variable analyses, JAGES Taketoyo study. J Epidemiol Community Health 69:905-910. https://doi. org/10.1136/jech-2014-205345 
44. Berkman LF, Glass T, Brissette I et al (2000) From social integration to health: Durkheim in the new millennium. Soc Sci Med 51:843-857. https://doi.org/10.1016/s0277-9536(00)00065-4

45. Huo YR, Suriyaarachchi P, Gomez F et al (2015) Comprehensive nutritional status in sarco-osteoporotic older fallers. J Nutr Health Aging 19:474-480. https://doi.org/10.1007/s12603-014-0543-Z

46. Chen Z, Jiang X, Shi G et al (2021) Social frailty and longitudinal risk of depressive symptoms in a Chinese population: the Rugao longevity and aging study. Psychogeriatrics 21:483-490. https:// doi.org/10.1111/psyg. 12696

47. Shankar A, McMunn A, Banks J et al (2011) Loneliness, social isolation, and behavioral and biological health indicators in older adults. Health Psychol 30:377-385. https://doi.org/10.1037/a0022 826

48. Dröes RM, Chattat R, Diaz A et al (2017) Social health and dementia: a European consensus on the operationalization of the concept and directions for research and practice. Aging Ment Health 21:4-17. https://doi.org/10.1080/13607863.2016.1254596

49. Kelley-Moore JA, Schumacher JG, Kahana E et al (2006) When do older adults become "disabled"? Social and health antecedents of perceived disability in a panel study of the oldest old. J Health Soc Behav 47:126-141. https://doi.org/10.1177/0022146506 04700203

50. Landeiro F, Leal J, Gray AM (2016) The impact of social isolation on delayed hospital discharges of older hip fracture patients and associated costs. Osteoporos Int 27:737-745. https://doi.org/10. 1007/s00198-015-3293-9

51. Ek S, Rizzuto D, Fratiglioni L et al (2019) Risk factors for injurious falls in older adults: the role of sex and length of follow-up. J Am Geriatr Soc 67:246-253. https://doi.org/10.1111/jgs.15657

52. Schrempft S, Jackowska M, Hamer M et al (2019) Associations between social isolation, loneliness, and objective physical activity in older men and women. BMC Public Health 19:74. https:// doi.org/10.1186/s12889-019-6424-y

Publisher's Note Springer Nature remains neutral with regard to jurisdictional claims in published maps and institutional affiliations. 